\section{Dann tut's dem Baby weniger weh}

Ärzte aus Kanada raten, bei Kindern im ersten Lebensjahr vor einer Impfung eine Lidocainsalbe auf die Einstichstelle zur Schmerzreduktion aufzutragen. Ein Video, das die Eltern anleitet, wie sie ihr Kind am besten halten und beruhigen, und eine orale Zuckerlösung hatten sich dagegen als wirkungslos erwiesen. Der Effekt der drei Interventionen war in einer Studie mit 352 gesunden Babys untersucht worden. Bei den Impfungen in den ersten zwölf Monaten waren je nach Gruppe entweder nur Placebo, nur der Anleitungsfilm, der Film plus eine $24 \%$ ige Zuckerlösung ( $2 \mathrm{ml}$, 1-2 min vor der Impfung) oder der Film plus Zucker plus eine 4\%ige Lidocainsalbe zum Einsatz gekommen. Die Schmerzen bei der Injektion, die anhand des Verhaltens der Kinder mit der Modified Behavioural Pain Scale (0-10) beurteilt wurden, erreichten 6,3 Punkte mit der Dreifachintervention, mit den anderen Maßnahmen waren es jeweils 6,8 Punkte.

Taddio A et al. Relative e.ectiveness of additive pain interventions during vaccination in infants. CMAJ 2017;189:E227-34

\section{Auch Väter mit „Babyblues“}

Die Schwangerschaft der Partnerin und die erste Zeit mit dem Baby setzen Väter unter Stress, wie eine neuseeländische Studie zeigt. 3.523 Männer, allesamt Partner von Teilnehmerinnen der "Growing Up in New Zealand"-Studie, wurden untersucht. 2,3\% von innen zeigten im letzten Schwangerschaftstrimester und 4,3\% neun Monate nach der Geburt Zeichen einer Depression. Die Raten der Frauen lagen bei $11,5 \%$ bzw. 8,0\%. Das Risiko wurde u.a. erhöht durch eine Depression in der Vorgeschichte (OR 2,80) und einen allgemein schlechten Gesundheitszustand (OR 1,69). Probleme mit der Partnerin, der Familie oder im Job kamen nach der Geburt oft erschwerend hinzu.

JAMA Psychiatry 2017; epub 15.2.17, doi: 10.1001/jamapsychiatry.2016.4234

\section{HBSC-Symposium - jetzt online}

Das Supplement zum 7. Heilberufe SCIENCE-Symposium auf dem Interprofessionellen Gesundheitskongress in Dresden (28.-29. April 2017) ist jetzt online.

www.link.springer.com

\section{HIV-Infizierte haben heute eine fast normale Lebenserwartung}

\author{
Die Lebenserwartung HIV-infizierter Menschen ist seit 1996 um zehn Jahre \\ gestiegen. Ein 20-Jähriger, der nun eine antiretrovirale Therapie beginnt, wird \\ schätzungsweise 74 Jahre alt.
}

A nalysen in 18 europäischen und nordamerikanischen Kohorten haben jetzt bestätigt, dass HIV-infizierte Menschen heute deutlich länger leben, als noch in den 1990er-Jahren oder zu Beginn dieses Jahrhunderts. Die Lebenserwartung kommt jener in der Gesamtbevölkerung sehr nahe, erreicht sie aber noch nicht ganz. Das berichtet die internationale Antiretroviral Therapy Cohort Collaboration (ART-CC). Der Forschungsverbund hat Daten von 88.504 HIV-Patienten ausgewertet, die zwischen 1996 und 2010 eine ART begonnen hatten. Demnach fiel die Gesamtmortalitätsrate bei HIV-Infizierten mit Therapiebeginn zwischen 2008 und 2010 um 29\% niedriger aus als bei Therapiebeginn zwischen 2000 und 2003. Dieser Erfolg war in der Gruppe jener Patienten, die intravenös Drogen konsumieren, geringer, bestätigte sich aber ansonsten in fast allen betrachteten Subgruppen, so Adam Trickey von der Universität Bristol in Großbritannien und seine Kollegen.

\section{Mit ART die Lebenserwartung erhöhen}

Zwischen 1996 und 2010 stieg die Lebenserwartung einer 20-jährigen Frau mit HIV, die eine ART beginnt, um neun Jahre, die eines 20-jährigen Mannes um zehn Jahre. In Europa werden diese Patienten schätzungsweise 68 Jahre alt, in Nordamerika etwa 66 Jahre, so die Berechnungen der Studienautoren. Bezieht man die CD4-Zellzahl ein, prognostizieren sie derzeit für einen 20 Jahre alten Patienten, der nach einem Jahr Therapie eine CD-4-Zellzahl von über 350/Mikroliter aufweist, eine Lebenserwartung von sogar 78 Jahren. Die Daten stützen zudem die seit 2015 empfohlene Praxis, stets bei allen HIV-Patienten die ART unmittelbar nach Diagnosestellung zu beginnen.

Diese günstige Entwicklung sei nicht allein auf die verbesserte Effektivität und
Verträglichkeit moderner antiretroviraler Medikamente zurückzuführen, meint das Autorenteam. Es müssen deutlich weniger Medikamente eingenommen und die Therapieregime sind einfacher als früher, die Virusreplikation wird besser verhindert und die Viren haben es schwerer, Resistenzen zu entwickeln. Treten doch Resistenzen auf, stehen verschiedene Alternativregime zur Verfügung. Die verringerte Toxizität führt zu einer besseren Therapieadhärenz, Screening- und Präventionsprogramme greifen. "Mit der Erfahrung, dass HIV-positive Menschen alt werden können, screenen Ärzte intensiver und behandeln Komorbiditäten aggressiver, besonders was kardiovaskuläre Krankheiten, Hepatitis C und Krebs angeht", schreiben Trickey und seine Kollegen. Die Patienten werden ermutigt aufzuhören zu rauchen und motiviert, die Therapie konsequent durchzuhalten.

Die positiven Resultate all dieser Bemühungen, schränken sie ein, treffen freilich nur zu auf entwickelte Länder und dort wiederum auf Patienten mit hohem sozioökonomischen Status. Unter diesen Umständen sei es unwahrscheinlich, dass die Sterberaten mit neuentwickelten Medikamenten noch wesentlich verringert werden könnten. „Jetzt müssen wir auf Fragen der Therapieadhärenz, der späten Diagnosestellung sowie die Diagnostik und Behandlung bei Komorbiditäten fokussieren", geben die Experten einen Ausblick. Bei Drogengebrauchern müsse die ART gefördert, der Zugang zur Therapie verbessert werden, fordert Trickey, besonders auch bei Hepatitis-C-Koinfektion. Denn die Mehrheit HIV-bedingter Todesfälle betrifft heute Menschen, die gar nicht behandelt werden.

(TM)

Trickey A et al. Survival of HIV-positive patients starting antiretroviral therapy between 1996 and The Lancet HIV, DOI: http://dx.doi.org/10.1016/ S2352-3018(17)30066-8; online 10. Mai 2017 2013: a collaborative analysis of cohort studies. 